\title{
Death-associated protein kinase 3 controls the tumor progression of A549 cells through ERK MAPK/c-Myc signaling
}

\author{
SATORU KAKE ${ }^{1,2^{*}}$, TATSUYA USUI ${ }^{3 *}$, TAKASHI OHAMA ${ }^{1}$, \\ HIDEYUKI YAMAWAKI ${ }^{4}$ and KOICHI SATO ${ }^{1}$ \\ ${ }^{1}$ Laboratory of Veterinary Pharmacology, Joint Faculty of Veterinary Medicine, Yamaguchi University, Yoshida, Yamaguchi; \\ ${ }^{2}$ Department of Comparative Animal Science, College of Life Science, Kurashiki University of Science and The Arts, \\ Kurashiki, Okayama; ${ }^{3}$ Laboratory of Veterinary Toxicology, Joint Faculty of Veterinary Medicine, \\ Yamaguchi University, Yoshida, Yamaguchi; ${ }^{4}$ Laboratory of Veterinary Pharmacology, \\ School of Veterinary Medicine, Kitasato University, Towada, Aomori, Japan
}

Received July 29, 2016; Accepted September 20, 2016

DOI: $10.3892 /$ or.2017.5359

\begin{abstract}
Death-associated protein kinases (DAPKs) are members of the serine/threonine protein kinase family, which regulate cell death. Although DAPK3 has been implicated as a tumor suppressor, a recent study revealed an oncogenic role of DAPK3. However, the role of DAPK3 in non-small cell lung cancer (NSCLC) remains unclear. Therefore, we examined whether DAPK3 controls the progression of NSCLC using the NSCLC cell line, A549. We generated A549 cells stably expressing small hairpin RNA (shRNA) targeting DAPK3. In the A549 cells, the protein level of DAPK3 was decreased and the cell proliferation was inhibited. DAPK 3 knockdown caused $\mathrm{G}_{1} / \mathrm{G}_{0}$ cell cycle arrest as assessed by flow cytometric assay and reduced cyclin D1 expression in A549 cells. Phosphorylation of ERK and c-Myc, but not Akt and JNK, was inhibited by DAPK3 knockdown. Cell migration and invasion were also inhibited by DAPK3 knockdown as determined by a Boyden chamber assay and an invasion assay, respectively. Moreover, DAPK3 knockdown inhibited anchorage-independent cell growth as determined by soft-agar colony formation assay. In a mouse xenograft model, tumors derived from DAPK3knockdown cells exhibited reduced tumor growth. The present results demonstrated for the first time that DAPK3 controls proliferation, migration, invasion, soft-agar colony formation and tumor growth through activation of ERK/c-Myc signaling
\end{abstract}

Correspondence to: Dr Tatsuya Usui, Laboratory of Veterinary Toxicology, Joint Faculty of Veterinary Medicine, Yamaguchi University, 1677-1 Yoshida, Yamaguchi 753-8515, Japan

E-mail: usui@yamaguchi-u.ac.jp

*Contributed equally

Key words: death-associated protein kinase, non-small cell lung cancer, signal transduction, small hairpin RNA, tumor growth in A549 cells. These findings indicate that DAPK3 may be a novel target for the treatment of NSCLC.

\section{Introduction}

Lung cancer is one of the most common types of cancer and the leading cause of cancer-related deaths worldwide $(1,2)$. Lung cancer is classified into two groups. One is small cell lung cancer and the other is non-small cell lung cancer (NSCLC). NSCLC includes squamous cell carcinoma, adenocarcinoma and large cell carcinoma, which account for $80-85 \%$ of all lung cancer cases (1). Although there has been a significant development in the therapies of NSCLC for decades, the 5-year survival rate is still less than $15 \%$ (3). Therefore, new therapeutic targets are required to improve survival and quality of life.

Death-associated kinase 3 (DAPK3) is a member of the DAPK family. The DAPK family also includes DAPK1 and DAPK2. These proteins contain a similar N-terminal kinase domain and play a role in the induction of cell death (4). Moreover, DAPK3 was found to mediate DAPK1-induced apoptosis in HEK 293T cells (5), and to promote starvationinduced autophagy, an alternate type of programmed cell death, through the regulation of Atg9-mediated autophagosome formation (6). Furthermore, the expression of DAPK3 was found to be decreased in several squamous cell carcinomas $(7,8)$. In view of this evidence, DAPK3 has been regarded as a tumor suppressor.

In contrast, a recent study showed that DAPK3 promotes cancer cell proliferation rather than the induction of apoptosis in various cancer cell lines. In prostate cancer cells, DAPK3 promoted cell proliferation through the activation of the androgen receptor (9). In gastric cancer cells, overexpression of DAPK3 propagated cell proliferation, migration and invasion via activation of Akt and $\mathrm{NF}-\kappa \mathrm{B}$ signals and promotion of stemness (8). Furthermore, knockdown of DAPK3 prevented cell proliferation in colon cancer cells through the inhibition of $\mathrm{Wnt} / \beta$-catenin signals (10). These studies suggest the possibility of DAPK 3 as a novel therapeutic target of cancer. 
In lung cancer tissues, the DAPK3 gene was heterozygously mutated at a frequency of $3.2 \%$ (11). Nevertheless, it remains to be clarified how the wild-type DAPK3 gene controls NSCLC pathogenesis through a signaling pathway. We therefore examined whether knockdown of the DAPK3 gene affects NSCLC progression via cellular signaling. To the very best of our knowledge, in the present study we demonstrated for the first time that the DAPK3 gene regulates cell proliferation, migration and invasion through ERK/c-Myc signaling in A549 cells. It was also established that the DAPK3 gene plays a role in the tumor growth of A549 cells in vivo.

\section{Materials and methods}

Materials. Antibody sources were as follows: phospho-ERK, phospho-Akt, total-ERK, total-JNK, total-Akt, totalc-Myc (Cell Signaling Technology, Inc., Danvers, MA, USA); phospho-c-Myc (Bio Academia, Inc., Osaka, Japan); phospho-JNK (Promega, Madison, WI, USA); total-VCP (GeneTex, Inc., Irvine, CA, USA); total-cyclin D1 (Bioss, Inc., Woburn, MA, USA). Total-DAPK3 was provided by Dr Tachibana (Osaka City University, Japan).

Cell culture. A549 (human lung adenocarcinoma) cells were kindly provided by Dr B. Shimizu (Yamaguchi University, Ube, Japan). A549 cells were cultured in Dulbecco's modified Eagle's medium (DMEM) supplemented with $10 \%$ fetal bovine serum (FBS; Invitrogen, Carlsbad, CA, USA).

Short hairpin (sh)RNA and lentivirus production. Human DAPK3 small hairpin RNA (shRNA) and shNon target (shNT) expressing cells were produced as previously described (12). Briefly, shRNA complementary DNA strands (shNT, 5'-CAACAAGATGAGAGCACCA-3'; shDAPK3, 5'-GACGG ACGTGGTCCTCATC-3') were annealed and ligated into MluI/ClaI sites of the pLV-mC vector (Takara, Tokyo, Japan). To produce lentiviruses, $1 \mu \mathrm{g}$ of $\mathrm{pLV}-\mathrm{mC}, 0.77 \mu \mathrm{g}$ of a packaging plasmid (psPAX2) and $0.43 \mu \mathrm{g}$ of a protein-coated plasmid expressing vesicular stomatitis virus $G$ protein (pMD2.G) were transfected into Lenti-X 293T cells using $2.5 \mu 1$ of PEI Max (Polysciences, Inc., Warrington, PA, USA) dissolved in $333 \mu \mathrm{l}$ of Opti-MEM (Invitrogen). After $48 \mathrm{~h}$, viral supernatants were collected and filtered. A549 cells were treated with them for $8 \mathrm{~h}$.

Cell proliferation analysis. Cells $\left(1 \times 10^{4}\right)$ were seeded on a 12 -well culture plate. Cell proliferation was examined by cell counting using Cell Counting Kit-8 (CCK-8; Dojindo, Kumamoto, Japan) as previously described $(13,14)$. The absorbance of the medium at $450 \mathrm{~nm}$ was read on a standard plate reader (Beckman Coulter DU-800; Beckman Coulter, Inc., Brea, CA, USA).

Flow cytometry. After A549 cells expressing DAPK3 shRNA or shNT were seeded at a density of $2 \times 10^{5}$ cells on a 6 -well plate and incubated for $24 \mathrm{~h}$, they were trypsinized and collected into a $1.5 \mathrm{ml}$ tube. After the cells were fixed with $70 \%$ ethanol for $20 \mathrm{~min}$ at $-20^{\circ} \mathrm{C}$, they were washed with HEPES-buffered saline (HBS) and treated with RNase Staining Solution $(200 \mu \mathrm{g} / \mathrm{ml}$; Cell Signaling) for $30 \mathrm{~min}$. Next, they were washed with HBS and stained with propidium iodide (50 $\mu \mathrm{g} / \mathrm{ml}$; Cell Signaling) for $30 \mathrm{~min}$ at room temperature in the dark. The cell cycle data were obtained using BD Accuri ${ }^{\mathrm{TM}}$ C6 (BD Biosciences, San Jose, CA, USA). In addition, the cell population data were analyzed using BD Accuri software.

Western blotting. Western blotting was performed as previously described (14). Protein lysates were obtained by homogenizing cells with Triton-based lysis buffer [50 mM Tris- $\mathrm{HCl}$ ( $\mathrm{pH} 8.0)$, $5 \mathrm{mM}$ EDTA, $5 \mathrm{mM}$ EGTA, $1 \%$ Triton X-100, $1 \mathrm{mM} \mathrm{Na} \mathrm{VO}_{4}$, $20 \mathrm{mM}$ sodium pyrophosphate and Roche Complete protease inhibitor mixture]. Loading proteins (20-40 $\mu \mathrm{g})$ were separated by SDS-PAGE (10\%) and transferred to a nitrocellulose membrane (Wako, Osaka, Japan). After being blocked with 3\% bovine serum albumin or $0.5 \%$ skim milk, the membranes were incubated with primary antibodies at $4^{\circ} \mathrm{C}$ overnight. Then, the membranes were treated with secondary antibodies $(1: 10,000$ dilution, $1 \mathrm{~h}$ ) and ECL Pro (PerkinElmer, Freiburg, Germany). The results were visualized using LAS-3000 (FujiFilm, Tokyo, Japan) and quantified using ImageJ densitometric analysis software (National Institutes of Health, Bethesda, MD, USA).

Boyden chamber and invasion assays. The Boyden chamber assay was performed as previously described $(12,15)$. After membranes were coated with $2 \%$ gelatin (for the Boyden chamber assay) or 2\% Matrigel (for the invasion assay), A549 cells expressing DAPK3 shRNA or shNT were seeded at a density of $1 \times 10^{4}$ cells in the upper chamber. To migrate the cells, $600 \mu \mathrm{l}$ of medium with $10 \%$ FBS was added to the lower chamber. The membranes to which the cells migrated or invaded were fixed with methanol for $15 \mathrm{~min}$ and stained with Giemsa solution. The numbers of migrated and invaded cells through the membranes were counted under a light microscope and averaged.

Colony formation assay. The colony formation assay was performed as previously described (12). Cells $\left(1 \times 10^{3}\right)$ were seeded on a $60-\mathrm{mm}$ dish and cultured for 1 week. After the cells were fixed with $99.5 \%$ ethanol, colonies were stained with Giemsa. The number of surviving colonies was counted.

Soft-agar colony formation assay. For the soft-agar colony formation assay, $2 \times 10^{3}$ cells were seeded in $1.5 \mathrm{ml}$ top agar (DMEM containing 10\% FBS, $2.8 \% \mathrm{NaHCO}_{3}, 1 \mathrm{X}$ antibiotic/antimicotic and $0.36 \%$ agarose) on a $2.5 \mathrm{ml}$ bottom layer of solidified bottom agar (DMEM containing 10\% FBS, $2.8 \%$ $\mathrm{NaHCO}_{3}, 1 \mathrm{X}$ antibiotic/antimicotic and $0.75 \%$ agarose) in a 6 -well culture plate and cultured for 3 weeks. Colonies were stained with crystal violet solution and the number of colonies was counted.

Mouse xenograft assay. NOD.CB17 mouse Prkdc scid $/ \mathrm{J}$ mice were obtained from Japan SLC (Hamamatsu, Japan). All studies involving mice were conducted according to the Guide to Animal Use and Care of the Yamaguchi University and approved by the Ethics Committee. After A549 cells expressing shDAPK3 or shNT were subcutaneously injected into the flanks of NOD/SCID mice (5-6 weeks, $1 \times 10^{6}$ cells each), the tumor volumes were measured every week for 6 weeks and estimated using the following formula: Tumor 
A

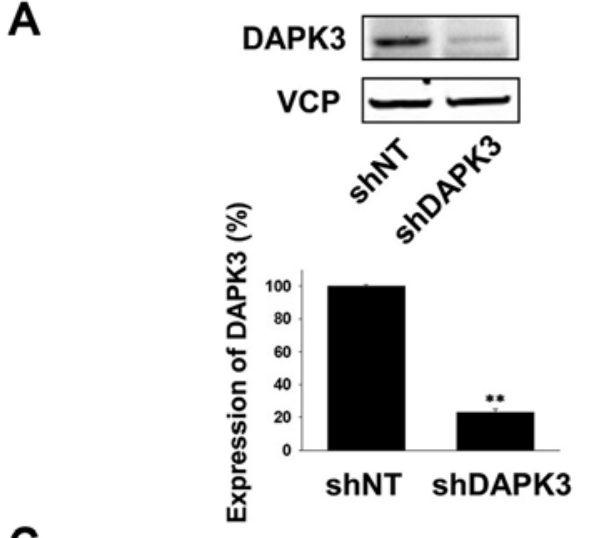

C

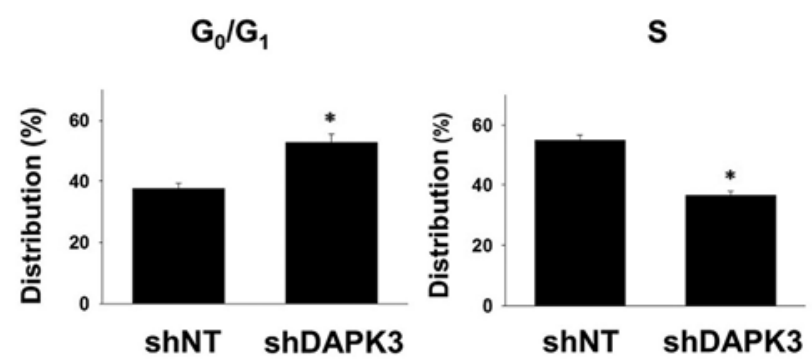

B

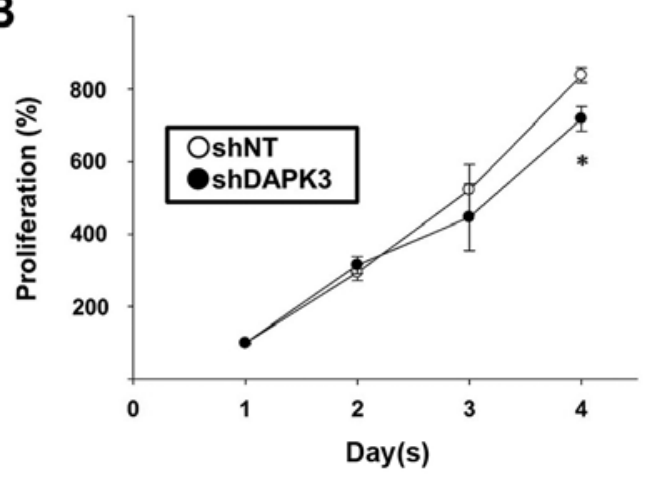

D

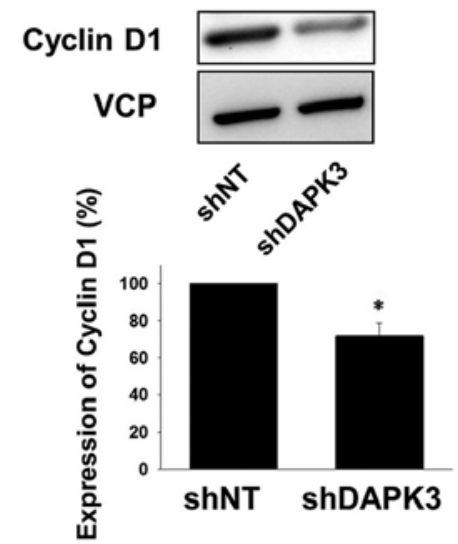

Figure 1. Effects of death-associated protein kinase (DAPK) 3 gene knockdown on the proliferation of A549 cells. Generation of DAPK3 short hairpin RNA (shRNA) stably expressing A549 cells. (A) After A549 cells were infected with the lentivirus of DAPK3 shRNA (shDAPK3) or non target shRNA $(\mathrm{shNT})$, the protein expression of DAPK3 was determined by western blotting $(\mathrm{n}=3)$. Equal protein loading was confirmed using a total VCP antibody; ${ }^{* *} \mathrm{P}<0.01$ vs. shNT. (B) After shNT or shDAPK3-expressing A549 cells were seeded into a 12 -well culture dish, the cell proliferation was evaluated by a cell counting assay for 4 consecutive days $(n=4)$. Cell proliferation is expressed as the mean $\pm \mathrm{SEM} ;{ }^{*} \mathrm{P}<0.05 \mathrm{vs}$. shNT. Effects of DAPK 3 gene knockdown on cell cycle arrest in A549 cells. After shNT- or shDAPK3-expressing A549 cells were collected, they were stained with cell cycle agents. Cell cycle progression was analyzed by flow cytometry $(n=4)$. (C) The percentages of A549 cells in the $\mathrm{G}_{0} / \mathrm{G}_{1}$ and $\mathrm{S}$ phase were expressed as the mean $\pm \mathrm{SEM}$; ${ }^{*} \mathrm{P}<0.05$ vs. shNT. (D) Effects of DAPK3 gene knockdown on the expression of a cell cycle regulatory protein. After shNT- or shDAPK3-expressing A549 cells were seeded, cyclin D1 protein expression was determined by western blotting $(\mathrm{n}=5)$. Equal protein loading was confirmed using a total VCP antibody; ${ }^{*} \mathrm{P}<0.05$ vs. shNT.

volume $=0.5 \times\left(\right.$ length $\mathrm{x}$ width $\left.{ }^{2}\right)$. Subsequently, the mice were euthanized, the tumor tissues were isolated and the tumor weights were assessed.

Statistical analysis. Data are shown as the means \pm SEM. Statistical evaluations were performed using Student's t-test between two groups. Values of $\mathrm{P}<0.05$ were considered statistically significant.

\section{Results}

Effects of DAPK3 gene knockdown on proliferation of A549 cells. Since several studies have shown that the DAPK3 gene is mutated in several types of cancer cells (11), we investigated whether DAPK3 is mutated in A549 cells. No DAPK3 mutation was observed in the A549 cells (data not shown). To clarify the role of the wild-type DAPK3 gene in NSCLC, we generated DAPK3 shRNA-expressing A549 cells. We confirmed that the expression of the DAPK3 protein was significantly decreased in the shDAPK3-expressing A549 cells compared with the shNTexpressing cells (Fig. 1A). In order to examine the effects of DAPK3 gene knockdown on A549 cell proliferation, we used a cell counting assay. The cell proliferation at day 4 was significantly lower in the DAPK3-knocked down cells compared with the proliferation of the shNT-expressing cells (Fig. 1B).

Effects of DAPK3 gene knockdown on cell cycle arrest in A549 cells. We next performed cell cycle analysis by flow cytometry. In the DAPK3-knockdown A549 cells, the number of cells in the $G_{0} / G_{1}$ phase was significantly increased and the number of cells in the $S$ phase was significantly decreased (Fig. 1C). We further examined the effects of DAPK3 gene knockdown on the expression of a cell cycle regulatory protein, cyclin D1, which regulates progression of the $G_{1}$ phase (16). Expression of cyclin D1 was significantly decreased in the DAPK3-knockdown A549 cells (Fig. 1D).

Effects of DAPK3 gene knockdown on proliferation-related signals in A549 cells. We next examined whether DAPK3 mediates the proliferation-related signals in A549 cells. Phosphorylation of ERK and c-Myc was significantly inhibited by DAPK3 gene knockdown (Fig. 2A and B). In contrast, DAPK3 gene knockdown had no effect on the phosphorylation of Akt and JNK (Fig. 2C and D). 
A

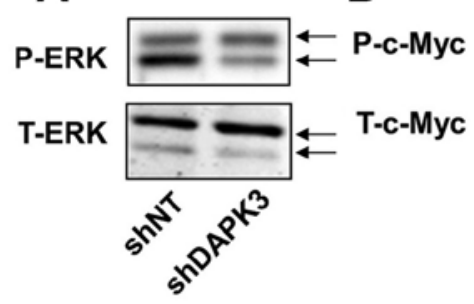

C
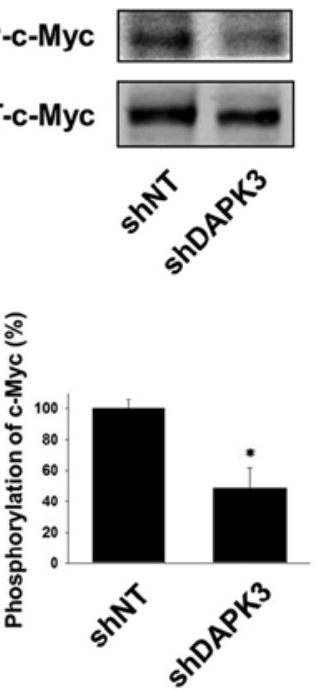
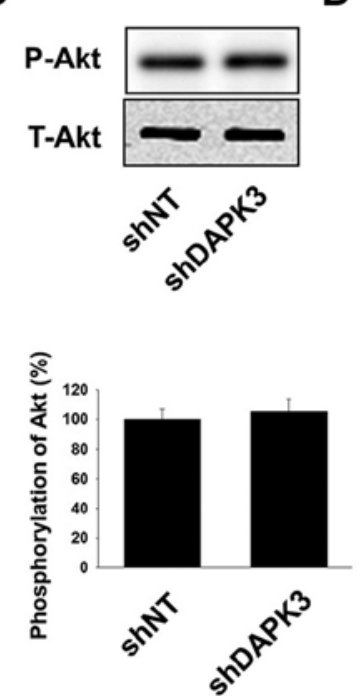

D
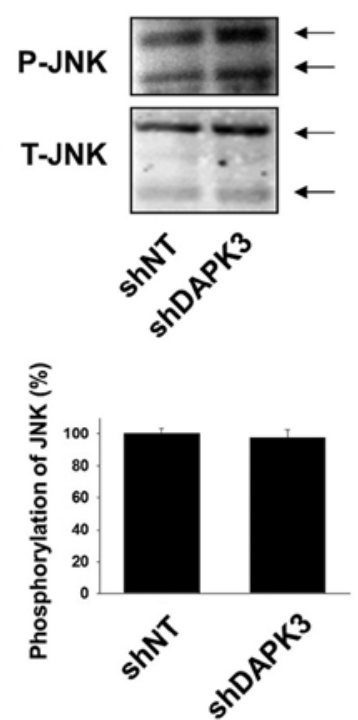

Figure 2. Effects of DAPK3 gene knockdown on proliferation-related signals in A549 cells. After shNT- or shDAPK3-expressing A549 cells were seeded, phosphorylation of $(\mathrm{A}, \mathrm{n}=4) \mathrm{ERK},(\mathrm{B}, \mathrm{n}=4) \mathrm{c}-\mathrm{Myc},(\mathrm{C}, \mathrm{n}=4)$ Akt and $(\mathrm{D}, \mathrm{n}=4) \mathrm{JNK}$ was determined by western blotting; ${ }^{*} \mathrm{P}<0.05$, ${ }^{* *} \mathrm{P}<0.01 \mathrm{vs}$. shNT. Arrows indicate the bands.

Effects of DAPK3 gene knockdown on migration, invasion and colony formation in A549 cells. Cell migration and invasion are essential for tumor metastasis (17). We thus examined the effects of DAPK3 gene knockdown on the migration and invasion of A549 cells. DAPK3 gene knockdown significantly inhibited cell migration (Fig. 3A) and invasion (Fig. 3B). Since cell colonization is also an important step in tumor metastasis (18), we examined the effects of DAPK3 gene knockdown on colony formation in A549 cells. Both anchorage-dependent colony formation (Fig. 4A) and anchorage-independent colony formation in soft-agar (Fig. 4B) were decreased by DAPK3 gene knockdown.

Effects of DAPK3 gene knockdown on tumor growth in a xenograft mouse model. We finally examined whether DAPK3 gene knockdown inhibits tumor growth in vivo. Injection of DAPK3 shRNA-expressing A549 cells into immunodeficient mice significantly slowed down tumor growth (Fig. 5A and B) and decreased tumor weight (Fig. 5C).

\section{Discussion}

In the present study, we examined whether DAPK3 mediates the tumor progression of A549 cells. The major findings of the present study are that the knockdown of the DAPK3 gene inhibited proliferation, cell cycle and activation of ERK and c-Myc (Figs. 1 and 2). It was also found that the knockdown of the DAPK3 gene inhibited migration and invasion (Fig. 3A and B). In addition, we showed that the knockdown of the DAPK3 gene inhibited colony formation (Fig. 4A and B). It was also shown that the knockdown of the DAPK3 gene slowed down tumor growth in a mouse xenograft model (Fig. 5). Collectively, our results indicate that DAPK3 mediates the tumor progression of A549 cells via cell proliferation, migration, invasion and colony formation through the activation of ERK/c-Myc signals.
In the present study, we showed that DAPK3 gene knockdown inhibited cell proliferation in A549 cells (Fig. 1). Since several studies have shown that DAPK3 has a pro-apoptotic effect on cancer cells (19), DAPK3 has been regarded as a tumor suppressor in various tumors. However, accumulating evidence has revealed that DAPK3 alternatively promotes cancer cell proliferation in some cancer cell lines. In human colon carcinoma cells, knockdown of the DAPK3 gene inhibited proliferation through the inhibition of $\mathrm{Wnt} / \beta$-catenin signaling (10). In gastric cancer cell lines, overexpression of DAPK3 promoted proliferation through the upregulation of Akt and NF- $\kappa \mathrm{B}$ signaling (8). The results of the present study concur with these studies. In the same study, the expression of DAPK 3 in primary gastric tumor tissues was lower compared with non-tumor tissues. However, the expression of DAPK3 increased in metastatic tissues compared with primary tumors. These clinical data suggest that DAPK3 may change into a tumor promoter from a tumor suppressor during the course of gastric tumor progression. Therefore, we speculate that DAPK3 also acquires a role as a metastatic promoter in the progression of NSCLC. Further clinical study on the correlation of DAPK3 expression and lung cancer metastasis may be required.

In NSCLC, KRAS mutations were observed at a frequency of $30 \%$ (20). KRAS mutations were also identified in A549 cells which are used in the present study (21). Mutated KRAS increases its activity and promotes cell proliferation through the interaction with Raf, which causes activation of MEK/ERK signaling (22). In the present study, we demonstrated that DAPK3 gene knockdown inhibited ERK activity in A549 cells (Fig. 2). Nevertheless, it was unclear how DAPK 3 controls ERK activity. In several cell lines, DAPK3 is regulated by DAPK1 (23) and a recent study showed that Raf interacts with DAPK1 in mitochondria, whose interaction increases reactive oxygen species (ROS) production (24). In addition, it was reported that inhibition of ROS prevented ERK 
A
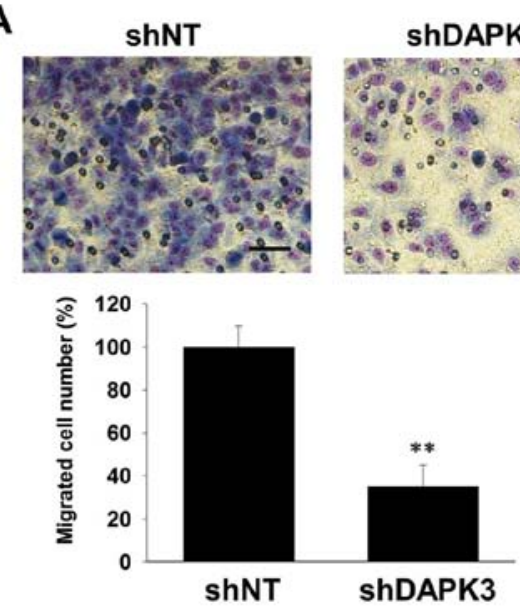

B

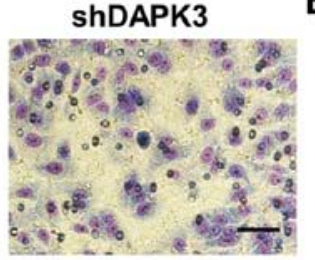

ShDAPK3
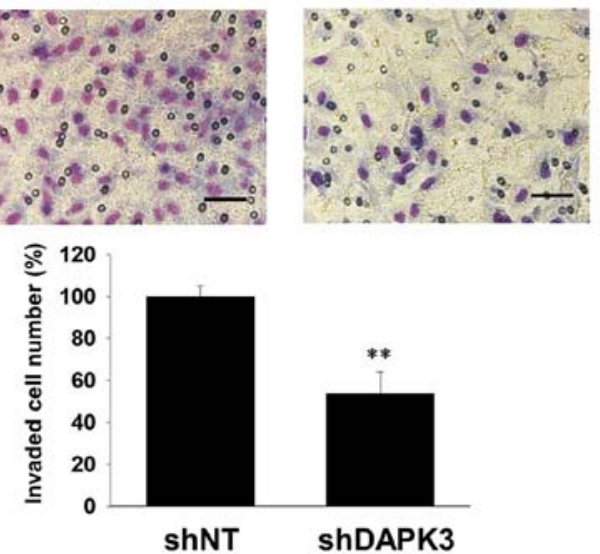

Figure 3. Effects of DAPK3 gene knockdown on migration and invasion in A549 cells. (A) Migration of A549 cells was determined by a Boyden chamber assay. Representative photomicrographs of A549 cells expressing shNT or shDAPK3 are shown. After the membranes were fixed with methanol, they were stained with Giemsa. Scale bar, $100 \mu \mathrm{m}$. The number of migrated cells was counted ( $\mathrm{n}=4) ;{ }^{* *} \mathrm{P}<0.01 \mathrm{vs.} \mathrm{shNT}$. (B) Invasion of A549 cells was determined by an invasion assay. Representative photomicrographs of A549 cells expressing shNT or shDAPK3 are shown. After the membranes were fixed with methanol, they were stained with Giemsa. Scale bar, $100 \mu \mathrm{m}$. The number of invaded cells was counted ( $\mathrm{n}=4) ;{ }^{* *} \mathrm{P}<0.01 \mathrm{vs}$. shNT.

A
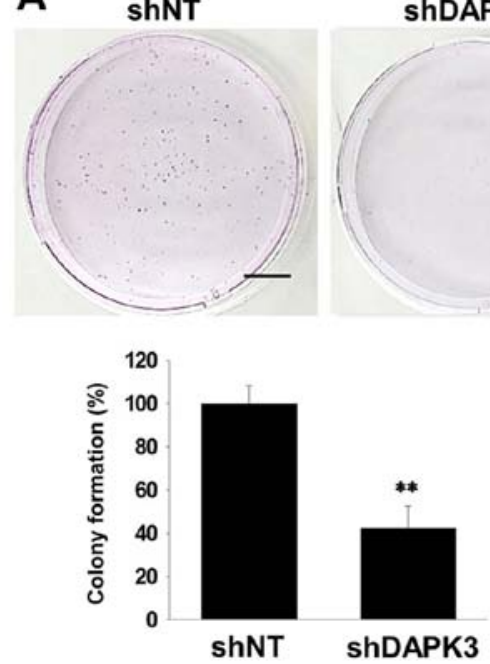

shDAPK3
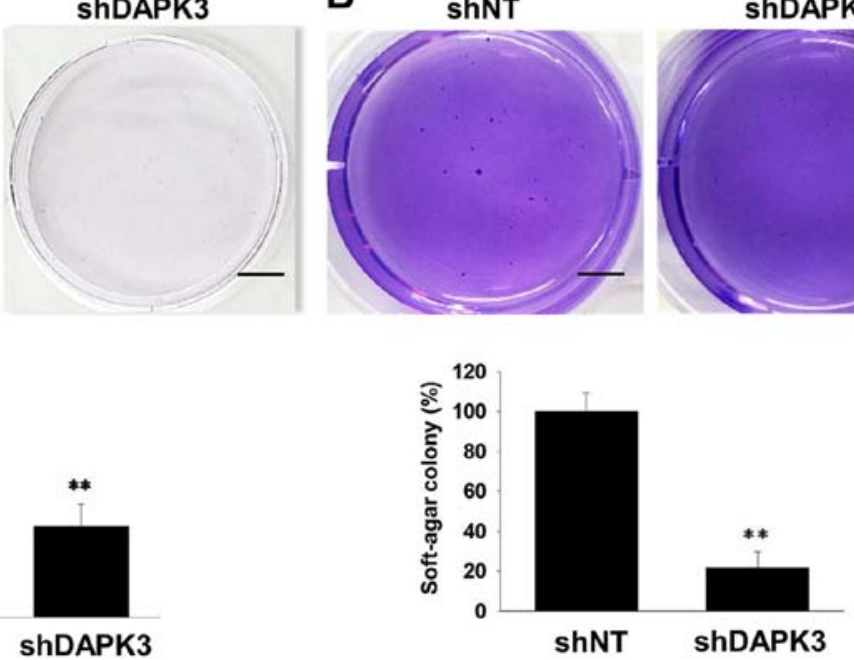

Figure 4. Effects of DAPK3 gene knockdown on colony formation in A549 cells. (A) Colony formation of A549 cells was determined by a colony formation assay. Representative photomicrographs of A549 cells expressing shNT or shDAPK3 are shown. After the membranes were fixed with $99.5 \%$ ethanol, colonies were stained with Giemsa. Scale bar, $1 \mathrm{~cm}$. The number of colonies was counted $(\mathrm{n}=4) ;{ }^{* *} \mathrm{P}<0.01 \mathrm{vs}$. shNT. (B) Anchorage-independent cell growth was determined by a soft agar colony formation assay. Representative photomicrographs of A549 cells expressing shNT or shDAPK3 are shown. The agar containing cells were stained with crystal violet. Scale bar, $5 \mathrm{~mm}$. The number of colonies was counted $(\mathrm{n}=4) ;{ }^{* *} \mathrm{P}<0.01 \mathrm{vs}$. shNT.

A

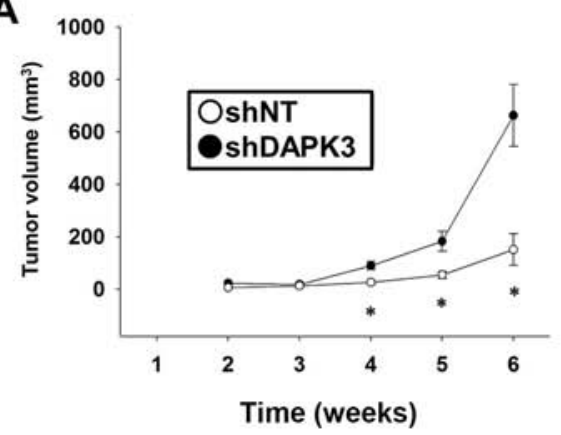

B

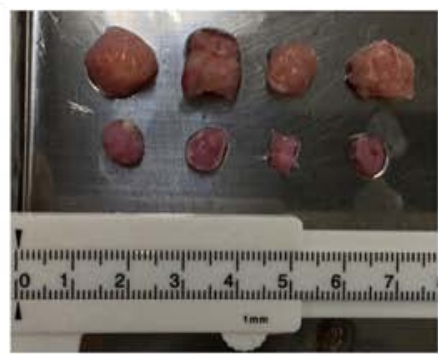

C

ShNT

ShDAPK3

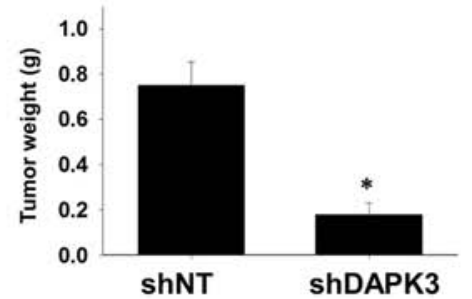

Figure 5. Effects of DAPK3 gene knockdown on tumor growth in a mouse xenograft model. After shNT- or shDAPK3-expressing A549 cells $\left(1 \times 10^{6}\right)$ were subcutaneously injected into the flanks of NOD/SCID mice, (A) the tumor volumes were measured by clipper every week for 6 weeks $(\mathrm{n}=4-5)$. (B) Images of shNT- or shDAPK3-expressing A549 tumors at the time of sacrifice (6 weeks post-injection) are shown. (C) The tumor weights $(n=4)$ were assessed and the results are expressed as the mean $\pm \mathrm{SEM} ;{ }^{*} \mathrm{P}<0.05$ vs. shNT. 
signaling in A549 cells (25). In the previous study, we showed that DAPK 3 promoted TNF- $\alpha$-induced activation of JNK, p38 and Akt through ROS generation (26). Considering these data, DAPK3 may mediate the interaction between Raf and DAPK1, which promotes ROS production and ERK activity in KRASmutated NSCLC cells.

Metastasis is responsible for the low 5-year survival rate of NSCLC (27). Migrated cancer cells gradually invade into blood or lymphatic vessels, which extravasate into distant tissue. In the previous study, we demonstrated that DAPK3 mediates platelet-derived growth factor BB-induced migration of vascular smooth muscle cells through p38/HSP27 signals (15). Another study showed that DAPK3 promoted migration and invasion of gastric cancer cell lines via activation of Akt and NF- $\kappa \mathrm{B}$ signals (8). In the present study, we found that DAPK3 gene knockdown inhibited migration and invasion of A549 cells (Fig. 3A and B). In addition, DAPK3 gene knockdown inhibited phosphorylation of ERK (Fig. 2A) but not p38 and NF- $\mathrm{BB}$ (data not shown) in A549 cells. Since ERK signaling regulates not only cell proliferation, but also migration and invasion of A549 cells (25), we hypothesize that DAPK3 mediates migration and invasion through ERK activity in the A549 cells. These data imply that DAPK3 plays a significant role in the metastasis of NSCLC.

Cancer stem cells have self-renewal potential and are able to form new tumors. A recent study showed that overexpression of DAPK3 increased colony formation of gastric cancer cells through the upregulation of stemness-related gene expression (8). In the present study, we showed that knockdown of the DAPK3 gene inhibited fossil formation and soft-agar colony formation (Fig. 4A and B). Although we did not investigate whether DAPK3 gene knockdown affects the expression of stemness-related genes in A549 cells, these results imply that DAPK3 regulates stemness of lung cancer cells, which leads to the formation of new tumors.

Although a previous study showed that overexpression of mutated DAPK3 promotes proliferation of lung cancer cells (11), it has not been determined whether DAPK3 mediates tumor growth in vivo. To the very best of our knowledge, in the present study we demonstrated for the first time that tumor growth of A549 cells was slowed down by DAPK3 gene knockdown in a mouse xenograft model (Fig. 5). While investigating whether DAPK3 is mutated in A549 cells, we observed no DAPK 3 mutation (data not shown). Therefore, we speculated that wild-type DAPK3 functions as an oncogene in the course of tumor growth in NSCLC. Further clinical investigations may be needed to confirm this theory.

In summary, to the very best of our knowledge, in the present study we demonstrated for the first time that DAPK3 controls proliferation, migration and invasion of A549 cells. It was also suggested that DAPK3 may be responsible for tumor growth and metastasis. Further studies on DAPK3 may contribute to the development of new therapies and knockdown of the DAPK3 gene may become a new strategy used in non-small lung cancer.

\section{Acknowledgements}

We thank Dr Tachibana and Dr Shimizu for kindly providing the DAPK3 antibody and A549 cells, respectively.

\section{References}

1. Jemal A, Siegel R, Ward E, Hao Y, Xu J and Thun MJ: Cancer statistics, 2009. CA Cancer J Clin 59: 225-249, 2009.

2. Torre LA, Bray F, Siegel RL, Ferlay J, Lortet-Tieulent J and Jemal A: Global cancer statistics, 2012. CA Cancer J Clin 65: 87-108, 2015.

3. Siegel R, Naishadham D and Jemal A: Cancer statistics, 2012. CA Cancer J Clin 62: 10-29, 2012.

4. Bialik S and Kimchi A: The death-associated protein kinases: Structure, function, and beyond. Annu Rev Biochem 75: 189-210, 2006.

5. Shani G, Marash L, Gozuacik D, Bialik S, Teitelbaum L, Shohat G and Kimchi A: Death-associated protein kinase phosphorylates ZIP kinase, forming a unique kinase hierarchy to activate its cell death functions. Mol Cell Biol 24: 8611-8626, 2004.

6. Tang HW, Wang YB, Wang SL, Wu MH, Lin SY and Chen GC: Atg1-mediated myosin II activation regulates autophagosome formation during starvation-induced autophagy. EMBO J 30: 636-651, 2011.

7. Mallipeddi R, Wessagowit V, South AP, Robson AM, Orchard GE, Eady RA and McGrath JA: Reduced expression of insulin-like growth factor-binding protein-3 (IGFBP-3) in Squamous cell carcinoma complicating recessive dystrophic epidermolysis bullosa. J Invest Dermatol 122: 1302-1309, 2004.

8. Li J, Deng Z, Wang Z, Wang D, Zhang L, Su Q, Lai Y, Li B, Luo Z, Chen X, et al: Zipper-interacting protein kinase promotes epithelial-mesenchymal transition, invasion and metastasis through AKT and NF-kB signaling and is associated with metastasis and poor prognosis in gastric cancer patients. Oncotarget 6: 8323-8338, 2015.

9. Leister P, Felten A, Chasan AI and Scheidtmann KH: ZIP kinase plays a crucial role in androgen receptor-mediated transcription. Oncogene 27: 3292-3300, 2008.

10. Togi S, Ikeda O, Kamitani S, Nakasuji M, Sekine Y, Muromoto R, Nanbo A, Oritani K, Kawai T, Akira S, et al: Zipper-interacting protein kinase (ZIPK) modulates canonical Wnt/beta-catenin signaling through interaction with Nemo-like kinase and T-cell factor 4 (NLK/TCF4). J Biol Chem 286: 19170-19177, 2011.

11. Brognard J,Zhang YW, Puto LA and Hunter T: Cancer-associated loss-of-function mutations implicate DAPK3 as a tumorsuppressing kinase. Cancer Res 71: 3152-3161, 2011.

12. Enjoji S, Yabe R, Fujiwara N, Tsuji S, Vitek MP, Mizuno T, Nakagawa T, Usui T, Ohama $T$ and Sato $K$ : The therapeutic effects of SET/I2PP2A inhibitors on canine melanoma. J Vet Med Sci 77: 1451-1456, 2015.

13. Usui T, Nijima R, Sakatsume T, Otani K, Kameshima S, Okada M and Yamawaki H: Eukaryotic elongation factor 2 kinase controls proliferation and migration of vascular smooth muscle cells. Acta Physiol 213: 472-480, 2015.

14. Yabe R, Miura A, Usui T, Mudrak I, Ogris E, Ohama T and Sato K: Protein phosphatase methyl-esterase PME-1 protects protein phosphatase $2 \mathrm{~A}$ from ubiquitin/proteasome degradation. PLoS One 10: e0145226, 2015.

15. Usui T, Sakatsume T, Nijima R, Otani K, Kazama K, Morita T, Kameshima S, Okada M and Yamawaki H: Death-associated protein kinase 3 mediates vascular structural remodelling via stimulating smooth muscle cell proliferation and migration. Clin Sci 127: 539-548, 2014.

16. Hunter T and Pines J: Cyclins and cancer. II: Cyclin D and CDK inhibitors come of age. Cell 79: 573-582, 1994.

17. Bravo-Cordero JJ, Hodgson L and Condeelis J: Directed cell invasion and migration during metastasis. Curr Opin Cell Biol 24: 277-283, 2012.

18. Scheel C and Weinberg RA: Cancer stem cells and epithelialmesenchymal transition: Concepts and molecular links. Semin Cancer Biol 22: 396-403, 2012.

19. Kawai T, Matsumoto M, Takeda K, Sanjo H and Akira S: ZIP kinase, a novel serine/threonine kinase which mediates apoptosis. Mol Cell Biol 18: 1642-1651, 1998.

20. Guin S, Ru Y, Wynes MW, Mishra R, Lu X, Owens C, Barn AE, Vasu VT, Hirsch FR, Kern JA, et al: Contributions of KRAS and RAL in non-small-cell lung cancer growth and progression. J Thorac Oncol 8: 1492-1501, 2013.

21. Oneyama C, Ikeda J, Okuzaki D, Suzuki K, Kanou T, Shintani Y, Morii E, Okumura M, Aozasa K and Okada M: MicroRNA-mediated downregulation of mTOR/FGFR3 controls tumor growth induced by Src-related oncogenic pathways. Oncogene 30: 3489-3501, 2011. 
22. An S, Yang Y, Ward R, Liu Y, Guo XX and Xu TR: A-Raf: A new star of the family of raf kinases. Crit Rev Biochem Mol Biol 50: 520-531, 2015

23. Endo A, Surks HK, Mochizuki S, Mochizuki N and Mendelsohn ME: Identification and characterization of zipperinteracting protein kinase as the unique vascular smooth muscle myosin phosphatase-associated kinase. J Biol Chem 279: 42055-42061, 2004

24. Tsai YT, Chuang MJ, Tang SH, Wu ST, Chen YC, Sun GH, Hsiao PW, Huang SM, Lee HJ, Yu CP, et al: Novel cancer therapeutics with allosteric modulation of the mitochondrial C-Raf-DAPK complex by Raf inhibitor combination therapy. Cancer Res 75: 3568-3582, 2015.
25. Ku MJ, Kim JH, Lee J, Cho JY, Chun T and Lee SY: Maclurin suppresses migration and invasion of human non-small-cell lung cancer cells via anti-oxidative activity and inhibition of the Src/FAK-ERK- $\beta$-catenin pathway. Mol Cell Biochem 402: 243-252, 2015

26. Usui T, Okada M, Hara Y and Yamawaki H: Death-associated protein kinase 3 mediates vascular inflammation and development of hypertension in spontaneously hypertensive rats. Hypertension 60: 1031-1039, 2012.

27. Marcus AI and Zhou W: LKB1 regulated pathways in lung cancer invasion and metastasis. J Thorac Oncol 5: 1883-1886, 2010. 